\title{
Follicular Thyroid Carcinoma Presenting as a Humeral Mass: A Rare Case Presentation
}

\author{
Marvin Jonne L. Mendoza ${ }^{1, \odot}$ \\ ${ }^{1}$ Division of Medical Oncology, Department of Medicine, University \\ of the Philippines-Philippine General Hospital, Taft Avenue, Ermita, \\ Manila, Philippines
}

Address for correspondence Marvin Jonne L. Mendoza, MD, Division of Medical Oncology, Department of Medicine, University of the Philippines-Philippine General Hospital, Taft Avenue, Ermita, Manila 1000, Philippines (e-mail: mlmendoza5@up.edu.ph).

\begin{abstract}
Follicular thyroid carcinoma is the second most common cancer of the thyroid, which usually presents as a solitary thyroid nodule. Diagnosis of follicular thyroid cancer involving the appendicular skeleton is very rare. In this paper, we report a case of a 65 -year-old woman who previously underwent partial lobectomy of the left thyroid gland, and who consequently presented with a 2-year history of gradually enlarging left arm mass. Initial whole-body bone imaging revealed a large osteolytic lesion in the humerus. An MRI (magnetic resonance imaging) of the left arm showed a heterogeneously enhancing, encapsulated, soft tissue mass with humeral involvement, and the initial impression was osteosarcoma versus fibrosarcoma. Biopsy reading of the humeral mass was morphologically compatible with a well-differentiated metastatic thyroid carcinoma. She subsequently underwent right lobectomy where histopathology showed Hashimoto's thyroiditis. A slide review was requested and the biopsy of the humeral mass revealed adenocarcinoma with follicular architecture, compatible with a metastatic thyroid carcinoma. Meanwhile, the findings on the right thyroid lobe revealed a multinodular colloid adenomatous goiter with hyperplastic features in a

Keywords

- follicular thyroid cancer

- osseous metastasis

- thyroid cancer background of Hashimoto's thyroiditis.

This report demonstrated a rare presentation of follicular thyroid carcinoma metastasizing to the humerus that can be confused with primary bone tumors, both clinically and radiologically. Early diagnosis and a high index of suspicion is foremost for immediate and appropriate management.
\end{abstract}

\section{Introduction}

Follicular thyroid cancer (FTC) is a well-differentiated type of thyroid malignancy and is the second most common thyroid cancer next to papillary carcinoma. ${ }^{1,2}$ It usually presents as a solitary thyroid nodule. Because of its propensity to spread hematogenously, distant metastases are more commonly encountered in FTC compared with the papillary type, ${ }^{3}$ with incidence rates between 11 and $25 \%{ }^{4}$ The most frequent sites of distant metastases are the lungs and bones. ${ }^{5}$ Osseous metastasis at initial presentation is rarely encountered and is associated with poor prognosis. ${ }^{2,5}$ In such scenarios, the axial skeleton particularly the spine, ribs, and pelvis are usually involved, resulting in symptoms such as severe pain and compression or pathologic fractures. ${ }^{2,6,7}$ Involvement of the appendicular skeleton is rarely reported in literature, and can often be confused with primary bone tumors. In this report, we discuss the unique case of a 65 -year-old female presenting with left humeral mass, diagnosed with metastatic thyroid carcinoma of follicular histology without a confirmed primary thyroid tumor.

\section{Case Presentation}

A 65-year-old woman with no known comorbidities presented with a 2-year history of left arm mass. Twenty-eight
DOI https://doi.org/ $10.1055 / \mathrm{s}-0040-1715763$ ISSN 2454-6798.
License terms

(1) (1) $\Theta \circledast$ 
years prior to consult, she presented with a gradually enlarging anterior neck mass associated with dysphagia for which she subsequently underwent partial lobectomy of the left thyroid gland. Histopathologic diagnosis of the resected mass was unknown to the patient and no further work ups were done. No other intervention was given to the patient.

In the interim, there were no subjective complaints until 2 years prior to consult when she noted a gradual enlargement of left arm mass associated with weakness, mild pain, and restriction in movement. A whole-body bone scan was done which showed a large osteolytic lesion affecting the mid shaft of the left humerus. There was also a note of a solitary mild focal retention at the left temporal area of the skull. Magnetic resonance imaging with contrast of the left arm revealed a heterogeneously enhancing, encapsulated, bulky, soft tissue mass, measuring approximately $15.4 \mathrm{~cm} \times 9.0 \mathrm{~cm} \times$ $10.0 \mathrm{~cm}(L \times W \times \mathrm{AP})$ with involvement of the diaphysis and metaphysis of the humerus exhibiting lysis of the adjacent bone. The differentials considered then were osteosarcoma and fibrosarcoma.

She consulted a local doctor and had a neck ultrasound which revealed a normal sized right thyroid lobe with parenchymal disease, and a note of a solid nodule in the mid to lower zone measuring $2.23 \mathrm{~cm} \times 1.60 \mathrm{~cm} \times 1.24 \mathrm{~cm}$, and a complex nodule in the upper to mid pole measuring $2.19 \mathrm{~cm} \times$ $1.69 \mathrm{~cm} \times 1.21 \mathrm{~cm}$. Ultrasound-guided fine needle aspiration revealed cytomorphologic findings suggestive of poorly differentiated carcinoma. On microscopic examination, the smears showed several scattered, highly anaplastic cells with bizarre, pleomorphic nuclei and fair amount of cytoplasm. She underwent lobectomy of the right thyroid gland 6 months prior to consult. Perioperative course was unremarkable. She was discharged on levothyroxine $75 \mu \mathrm{g}$ once a day, which she only took for 2 months. The final histopathological report then revealed Hashimoto's thyroiditis.

Three months prior to consult, she noted an increase in the size of the left arm mass. An orthopaedic surgeon did a core needle biopsy which revealed a well-differentiated meta-static thyroid carcinoma. She was referred to an endocrinologist for further assessment and management, but due to the size of the mass and the previous histopathology report showing a poorly differentiated subtype, radioactive iodine treatment was not recommended. She was referred to the Cancer Institute for further management. At the Cancer Institute, she was seen with normal physical examination findings except for a surgical scar at the neck and the large mass at the left upper arm, which limits the mobility and strength of the extremity. There were no signs of hypothyroidism.

A review of the humeral mass' histopathological slides revealed adenocarcinoma with follicular architecture, compatible with a metastatic thyroid carcinoma. Findings on the right thyroid lobe showed a multinodular colloid adenomatous goiter with hyperplastic features in a background of Hashimoto's thyroiditis. There was no definite malignancy identified. Immunohistochemistry study with thyroglobulin was recommended for further evaluation of the humeral mass. A 131-iodine whole body scan to test uptake, as well as thyroid function tests, were also ordered. She was prescribed with levothyroxine at $50 \mu \mathrm{g}$ once a day and bisphosphonate treatment was planned.

\section{Discussion}

Thyroid carcinoma is the most common malignancy of the endocrine system, ${ }^{1}$ representing 567,000 cases worldwide, with a global incidence rate of $3.1 \%$. The global incidence rate in women is reported to be three times higher than in men, and it represents $5.1 \%$ of the total estimated female cancer burden in 2018. The etiology of thyroid cancer is not well understood, and the only established risk factor for thyroid cancer is exposure to ionizing radiation. Literature reports that cigarette smoking, obesity, hormonal exposures, and environmental factors may also play a role. ${ }^{8}$ Prognostic factors include age at diagnosis, gender, size of the lesion, presence of capsular and/or vascular invasion, and distant metastases. ${ }^{9}$

FTC, along with the papillary variant, belongs to the well-differentiated type of thyroid malignancies. They comprise 10 to $15 \%$ of the total thyroid cancers. ${ }^{1}$ Compared with other malignancies FTC has a good prognosis. However, FTC is reported to be more aggressive compared with the papillary histology, ${ }^{1}$ and is also frequently observed in the older population. Distant metastasis typically occurs via the hematogenous route, with lungs, bone, brain, liver, and lymph nodes as the most common sites. ${ }^{1}$ When osseous spread occurs, the axial skeleton is frequently affected, with very rare involvement of the appendicular bones. The vertebrae are the most common site of bone metastasis, followed by the ribs, pelvis, skull, and rarely, the femur and humerus. ${ }^{1}$ The propensity for the axial bones is believed to be secondary to the blood flow distribution characteristics and the bone microenvironment, which includes growth factors favorable for tumor growth. ${ }^{10}$ Previous reports confirmed that only $4 \%$ of all thyroid cancer patients will present initially with bone metastasis and a silent primary tumor, similar to this case. ${ }^{1,11}$

Due to the unusual presentation of FTC in this case, the patient was initially considered to have a primary bone tumor. The primary symptoms for metastatic bone disease such as pain and pathological fracture were not prominent but rather, a large soft tissue mass on the left arm was noted. This presentation was rather unusual for metastasis and was more suggestive of primary bone malignancies.

Metastatic tumors are the most common malignancy involving the bone, ${ }^{5}$ and can be lytic, blastic, or mixed. Lytic lesions are frequently observed in liver, breast, and thyroid primary cancers. ${ }^{1}$ Similar to the case presented, osteolysis and blow out appearances are the usual picture of bone metastasis from a thyroid primary tumor. Unexpectedly, there were no malignant cells identified in the histopathological review of the thyroid specimen post lobectomy. Metastasis without primary thyroid gland tumor is a rare scenario. ${ }^{5}$ It is possible though that her first partial thyroid lobectomy was able to effectively remove the primary site of FTC, and symptoms have just manifested at a later time due to the indolent nature 
of well-differentiated thyroid cancer subtypes. Further studies are needed to understand the nature of the metastatic pattern of differentiated thyroid carcinomas.

Presence of distant metastasis in FTC decreases the quality of life and confers poor prognosis. ${ }^{1}$ However, with early diagnosis and high index of suspicion, along with appropriate management, survival rates may be improved. If managed accordingly, survival rates can increase up to $43 \%{ }^{11}$

\section{Conclusion}

This report highlighted the unusual presentation of a metastatic thyroid carcinoma involving the appendicular skeleton. In such similar cases, early diagnosis is foremost for better prognosis and appropriate and timely intervention.

\section{Conflict of Interest}

None declared.

\section{References}

1 Kar A, Naik S, Behera P, et al. Solitary metastasis to humerus: Unusual presentation of follicular thyroid carcinoma diagnosed by fine needle aspiration cytology. Int J Med Res Prof. 2016;2(5):212-215

2 Yang W, Cho W, Das S, Conboy P. Follicular thyroid carcinoma presenting with pathological fracture of the humerus at initial diagnosis. J Surg Case Rep 2017;2017(1):1-3
3 Stephen J, Thomas MB, Thomas MB. A case of papillary thyroid cancer with soft tissue metastasis. Int Surg J. 2018;5(10):3425-3429

4 Sevinc A, Buyukberber S, Sari R, Baysal T, Mizrak B. Follicular thyroid cancer presenting initially with soft tissue metastasis. Jpn J Clin Oncol 2000;30(1):27-29

5 Boz A, Tazegul G, Bozoglan H, et al. Bone metastases without primary tumor: a well-differentiated follicular thyroid carcinoma case. J Cancer Res Ther 2018;14(2):447-450

6 Kallel F, Hamza F, Charfeddine S, et al. Clinical features of bone metastasis for differentiated thyroid carcinoma: a study of 21 patients from a Tunisian center. Indian J Endocrinol Metab 2014;18(2):185-190

7 Pittas AG, Adler M, Fazzari M, et al. Bone metastases from thyroid carcinoma: clinical characteristics and prognostic variables in one hundred forty-six patients. Thyroid 2000;10(3):261-268

8 Thun M, Linet M, Cerhan J, et al. Cancer Epidemiology and Prevention, 4th ed. New York: Oxford University Press; 2018: 839-860

9 Ban EJ, Andrabi A, Grodski S, Yeung M, McLean C, Serpell J. Follicular thyroid cancer: minimally invasive tumours can give rise to metastases. ANZ J Surg 2012;82(3):136-139

10 Muresan MM, Olivier $P$, Leclère J, et al. Bone metastases from differentiated thyroid carcinoma. Endocr Relat Cancer 2008;15(1):37-49

11 Shaha AR, Shah JP, Loree TR. Differentiated thyroid cancer presenting initially with distant metastasis. Am J Surg 1997; 174(5):474-476 